\title{
Inhibition of mTOR induces autophagy and reduces toxicity of polyglutamine expansions in fly and mouse models of Huntington disease
}

\author{
Brinda Ravikumar ${ }^{1,6}$, Coralie Vacher ${ }^{1,6}$, Zdenek Berger $^{1,2}$, Janet E Davies ${ }^{1}$, Shouqing Luo ${ }^{1}$, Lourdes G Oroz ${ }^{1}$, \\ Francesco Scaravilli ${ }^{3}$, Douglas F Easton ${ }^{4}$, Rainer Duden ${ }^{5}$, Cahir J O’Kane ${ }^{2}$ \& David C Rubinsztein ${ }^{1}$
}

Huntington disease is one of nine inherited neurodegenerative disorders caused by a polyglutamine tract expansion. Expanded polyglutamine proteins accumulate abnormally in intracellular aggregates. Here we show that mammalian target of rapamycin (mTOR) is sequestered in polyglutamine aggregates in cell models, transgenic mice and human brains. Sequestration of mTOR impairs its kinase activity and induces autophagy, a key clearance pathway for mutant huntingtin fragments. This protects against polyglutamine toxicity, as the specific mTOR inhibitor rapamycin attenuates huntingtin accumulation and cell death in cell models of Huntington disease, and inhibition of autophagy has the converse effects. Furthermore, rapamycin protects against neurodegeneration in a fly model of Huntington disease, and the rapamycin analog $\mathrm{CCl}-779$ improved performance on four different behavioral tasks and decreased aggregate formation in a mouse model of Huntington disease. Our data provide proof-ofprinciple for the potential of inducing autophagy to treat Huntington disease.

Huntington disease is an autosomal dominant neurodegenerative condition caused by a $(\mathrm{CAG})_{n}$ expansion mutation $(n>35)$, which is translated into an abnormally long polyglutamine tract at the $\mathrm{N}$ terminus of huntingtin. There is no effective treatment for Huntington disease. Huntington disease and related diseases associated with polyglutamine expansions (spinocerebellar ataxia types 1, 2, 3, 6, 7 and 17; spinobulbar muscular atrophy and dentatorubral-pallidoluysian atrophy) are probably caused by gain-of-function mechanisms. The mutant proteins causing these diseases accumulate in intraneuronal aggregates (also called inclusions) ${ }^{1}$. Some propose that aggregates are deleterious, but others argue that aggregates are protective, which accounts for the partial discordance between the presence of aggregates in neuronal subtypes and the specific brain regions affected in Huntington disease ${ }^{2-4}$. No protective mechanisms for aggregates have been reported.

Polyglutamine-expanded proteins interact with various targets, including several transcription factors or cofactors, leading to dysregulation of certain transcriptional pathways. Polyglutamine diseases may be 'transcriptionopathies', but the transcriptional pathways affected may differ in specific diseases ${ }^{5-7}$.

Mutant huntingtin is cleaved to form $\mathrm{N}$-terminal fragments comprising the first 100-150 residues containing the polyglutamine repeats, which are believed to be the toxic species found in aggregates ${ }^{1}$.
Accordingly, the pathogenesis of Huntington disease is frequently modeled with exon 1 fragments, which cause toxicity and aggregate in cell models and in vivo.

The turnover of mutant huntingtin fragments and other aggregateprone proteins is impaired by inhibitors of the autophagy-lysosome pathway in cell lines ${ }^{8,9}$. To test whether induction of autophagy protected against the toxicity of mutant huntingtin, we previously treated cells with rapamycin, a specific inhibitor of $\mathrm{mTOR}^{10}$. mTOR is a kinase that regulates important cellular processes ${ }^{10}$ (Supplementary Fig. 1 online), and its activity inhibits autophagy in cells from yeast to human ${ }^{10}$. Treatment with rapamycin for $15 \mathrm{~h}$, starting $9 \mathrm{~h}$ after transfection with mutant huntingtin exon 1, reduced aggregate formation and cell death, consistent with the induction of autophagy. But cells had many more aggregates $33 \mathrm{~h}$ after transfection than $9 \mathrm{~h}$ after transfection with the same constructs, and treatment with rapamycin for $15 \mathrm{~h}$ starting at $33 \mathrm{~h}$ after transfection did not decrease aggregation or cell death. Thus, we concluded that the ability of rapamycin to inhibit mTOR activity might be impaired after prolonged huntingtin expression or increased aggregate formation ${ }^{8}$. Therefore, early treatment with rapamycin may attenuate Huntington disease in vivo, as rapamycin (and its analogs) are lipophilic, show good blood-brain barrier penetration ${ }^{11-14}$ and are designed for long-term use in humans ${ }^{11}$.

${ }^{1}$ Department of Medical Genetics, Cambridge Institute for Medical Research, Wellcome/MRC Building, Addenbrooke's Hospital, Hills Road, Cambridge CB2 2XY, UK.

${ }^{2}$ Department of Genetics, Cambridge University, Cambridge CB2 3EH, UK. ${ }^{3}$ Division of Neuropathology, Institute of Neurology, University College London, London, UK. ${ }^{4}$ Cancer Research U.K., Genetic Epidemiology Unit, Department of Public Health, University of Cambridge, Cambridge, UK. ${ }^{5}$ Department of Clinical Biochemistry, Cambridge Institute for Medical Research, Wellcome/MRC Building, Addenbrooke's Hospital, Hills Road, Cambridge, CB2 2XY, UK. ${ }^{6}$ These authors contributed equally to this work. Correspondence should be addressed to D.C.R. (dcr1000@cus.cam.ac.uk). 


\section{RESULTS}

mTOR is sequestered into huntingtin aggregates

We speculated that the ability of rapamycin to inhibit mTOR activity might become impaired after prolonged huntingtin expression if aggregates sequester and inactivate mTOR. mTOR was diffusely distributed in untransfected cells, in cells expressing wild-type protein $\left(\mathrm{Gln}_{23}\right.$; Fig. 1a) and in cells expressing mutant protein $\left(\mathrm{Gln}_{74}\right)$ without aggregates but colocalized with both cytoplasmic and nuclear aggregates in $>98 \%$ of mutant cells with aggregates (Fig. 1a and Supplementary Fig. 2 online). This degree of mTOR colocalization is much greater than observed with most other proteins previously analyzed ${ }^{15}$. We never observed mTOR aggregation in the absence of huntingtin aggregates (data not shown). Sequestration of mTOR was not an artefact of protein overexpression or aggregation, as we found no colocalization of certain upstream mTOR regulators or unrelated proteins (Akt, Pdk1, Pten) with mutant huntingtin aggregates (data not shown). mTOR was also sequestered in huntingtin aggregates in brains of transgenic mice expressing N-terminal mutant huntingtin fragments ${ }^{16}$ (Fig. 1b) and of individuals with Huntington disease (Fig. 1c), but not in brains of controls.
Western blots of whole-cell lysates showed that a substantial amount of mTOR (normally a $289-\mathrm{kDa}$ protein) was present in the form of an abnormally slowly migrating, high-molecular-mass product in the stacking gel (characteristic of aggregates) in mutant but not wild-type cells (Fig. 1d,e). The presence of mutant huntingtin in the stacking gel is frequently considered to be indicative of its insolubility ${ }^{17}$. We also detected this high-molecular-mass product in the stacking gel using antibodies to phosphorylated mTOR (Fig. 1e) and to green fluorescent protein (GFP) directed to the huntingtin transgene (Fig. 1e). Substantial amounts of endogenous mTOR in the mutant lines were sequestered into the insoluble fraction (Fig. 1d). We blotted lysates from these cell lines with numerous antibodies $(>20)$, including antibodies to hsp70, hdj1, CBP and other proteins that colocalize with aggregates, and never observed such as obvious accumulation of these proteins in the stacking gels (data not shown). Therefore, this interaction might be very tight.

We confirmed the interaction between mutant huntingtin and mTOR by coimmunoprecipitation of mTOR with mutant huntingtin exon 1 from PC12 and COS-7 cells, mostly as high-molecular-mass aggregates in the stack (Fig. 1f). The difference in the mobility of these high-molecular-mass immunoprecipitates in PC12 and COS-7 cells may reflect greater heterogeneity in aggregates in the transiently transfected cells versus the stable inducible cells. This is not a generic feature of mutant huntingtin, as similar immunoprecipitations did not pull down

Figure $1 \mathrm{mTOR}$ is sequestered in mutant huntingtin aggregates in cell models, transgenic mice and human brain. (a) COS-7 cells expressing EGFP-tagged wild-type ( $\mathrm{Gln}_{23} ; \mathrm{Q} 23$ ) or mutant $\left(G \ln _{74} ; \mathrm{Q} 74\right)$ huntingtin exon 1 (green) for $48 \mathrm{~h}$ stained for endogenous mTOR (red) show mTOR in mutant aggregates. (b) Doublelabeling immunohistochemistry of huntingtin (EM48; green) and mTOR (red) in brain tissue of mice expressing mutant huntingtin ${ }^{16}$.

(c) Immunohistochemistry of brain tissue from individuals with grade III Huntington disease or unaffected controls using antibody to mTOR. (d) Western blot of lysates from stable inducible PC12 cells $48 \mathrm{~h}$ after expression of wild-type $\left(\mathrm{Gln}_{23} ; \mathrm{Q} 23\right)$ or mutant ( $\left.\mathrm{IIn}_{74} ; \mathrm{Q} 74\right)$ huntingtin showing mTOR in the stacking gel (mTOR insoluble) in cells expressing mutant huntingtin. (e) High-molecular-mass aggregates of mTOR in both PC12 (i) and COS-7 (ii) cells expressing mutant ( $\mathrm{Gln}_{74} ; \mathrm{Q74}$ ) huntingtin. Blots of cell lysates from PC12 cells were probed with antibodies to phosphorylated mTOR (Ser2448; p-mTOR; iii) and to GFP (iv); both showed insoluble material in the stack. (f) Cell lysates from stable inducible PC12 cells and transiently transfected COS-7 cells expressing wild-type $\left(\mathrm{Gln}_{23} ; \mathrm{Q} 23\right)$ or mutant $\left(\mathrm{Gln}_{74} ; \mathrm{Q74}\right)$ huntingtin for $48 \mathrm{~h}$ were immunoprecipitated using antibody to GFP and immunoblotted with antibody to mTOR. Uninduced PC12 cells with mutant huntingtin (UI) and untransfected COS-7 cells (UT) were controls. (g) Brain lysates from mice expressing mutant huntingtin $(\mathrm{Tg})^{16}$ or control (Cont) littermates were immunoprecipitated with antibody to mTOR and immunodetected with an antibody recognizing the polyglutamine tract (IC2). Htt, huntingtin. 
proteins such as S6 kinase-1 (S6K1) and eIF-4E binding protein (4E$\mathrm{BP} 1$; data not shown). This interaction requires expanded polyglutamines, as wild-type huntingtin did not associate with mTOR. The mutant huntingtin fragment from brain lysates of transgenic mice also immunoprecipitated with antibody to mTOR (Fig. 1g).

Sequestration of mTOR to aggregates was associated with reduced amounts of soluble mTOR (relative to tubulin) in stable inducible cell lines expressing mutant versus wild-type huntingtin (Figs. 1d and 2a) and in brain lysates of mice expressing mutant huntingtin ${ }^{16}$ (Fig. 2b) versus control littermates. mTOR sequestration would impair nucleocytoplasmic shuttling of mTOR, which is crucial for its activity ${ }^{18}$.

\section{Polyglutamine expansion impairs mTOR kinase activity}

mTOR phosphorylates 4E-BP1 and ribosomal protein S6K1 (ref. 19), important regulators of cap-dependent and terminal oligopyrimidine tract (TOP)-dependent translation, respectively. 4E-BP1 was diffusely distributed in wild-type cells (Fig. 2c) but colocalized with aggregates in $\sim 30 \%$ of mutant cells with aggregates (Fig. 2 c) and was seen in nuclear inclusions of brains of individuals with Huntington disease (Fig. 2d). 4E-BP1 was not immunoprecipitated by mutant huntingtin, and so these interactions may be weak or transient. Unfortunately, we could not test whether S6K1 was localized to aggregates because the S6K1 antibody gave no specific immunocytochemical staining above

Figure 2 Reduced levels of soluble mTOR and impaired mTOR-dependent phosphorylation of its substrates S6K1 and 4E-BP1 in Huntington disease. (a) Reduced levels of soluble mTOR (as a function of tubulin) in stable inducible PC12 cells expressing mutant $\left(\mathrm{G} / \mathrm{n}_{74} ; \mathrm{Q} 74\right)$ huntingtin compared with those expressing wild-type $\left(\mathrm{Gln}_{23}\right.$; Q23) huntingtin (four independent experiments; $P$ $<0.0001$; unpaired $t$-test). (b) Reduced levels of soluble $\mathrm{mTOR}$ in brain lysates from mice expressing mutant huntingtin ${ }^{16}(\mathrm{M} 1, \mathrm{M} 2 ; n=9)$ compared with age-matched wild-type littermates (C1, C2; $n=9 ; P<0.001$, unpaired $t$-test). (c) COS-7 cells transiently transfected with wildtype ( $\left.\mathrm{Gln}_{23} ; \mathrm{Q} 23\right)$ or mutant $\left(\mathrm{Gln}_{74} ; \mathrm{Q74}\right)$ huntingtin (green) labeled for endogenous 4E-BP1 (red). (d) Immunohistochemistry of brain tissue from individuals with grade III Huntington disease showed $4 \mathrm{E}-\mathrm{BP} 1$ in intranuclear inclusions. No 4EBP1-stained inclusions were seen in controls (data not shown). (e) Western blot of cell lysates from stable inducible PC12 cells transfected with wildtype ( $\mathrm{Gl} \mathrm{n}_{23} ; \mathrm{Q} 23$ ) or mutant ( $\mathrm{Gl} \mathrm{n}_{74} ; \mathrm{Q74}$ ) huntingtin shows that cells expressing mutant huntingtin had $31 \%( \pm 4.3 \%$ s.e. $)$ less phosphorylated S6K1 (top) relative to total S6K1 (bottom) than cells expressing wild-type huntingtin. (f) Similarly, cells expressing mutant $\left(\mathrm{G} \ln _{74} ; \mathrm{Q} 74\right.$ ) huntingtin had $30 \%$ ( $\pm 4.5 \%$ s.d.) less phosphorylated 4E-BP1 (top) relative to total 4E-BP1 (bottom) than cells expressing wild-type $\left(\mathrm{G} \mathrm{In}_{23} ; \mathrm{Q} 23\right)$ huntingtin. Expression of wild-type and mutant huntingtin was induced for $48 \mathrm{~h}$ (e,f). (g) Cells expressing mutant ( $\left.\mathrm{Gln}_{74} ; \mathrm{Q74}\right)$ huntingtin also had lower levels of phosphorylated forms of transiently transfected FLAG-tagged 4EBP1 than cells expressing wild-type $\left(\mathrm{Gln}_{23} ; \mathrm{Q} 23\right)$ huntingtin. The multiple bands in $4 \mathrm{E}-\mathrm{BP} 1(\mathbf{f}, \mathbf{g})$ are due to its different phosphorylated species.

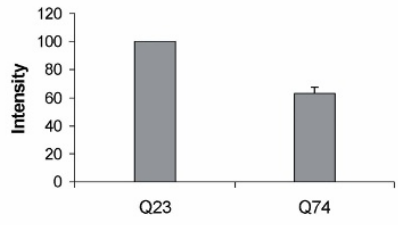

b

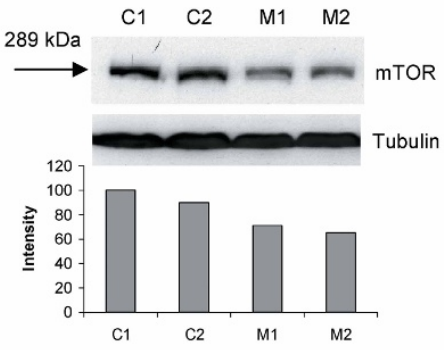

C
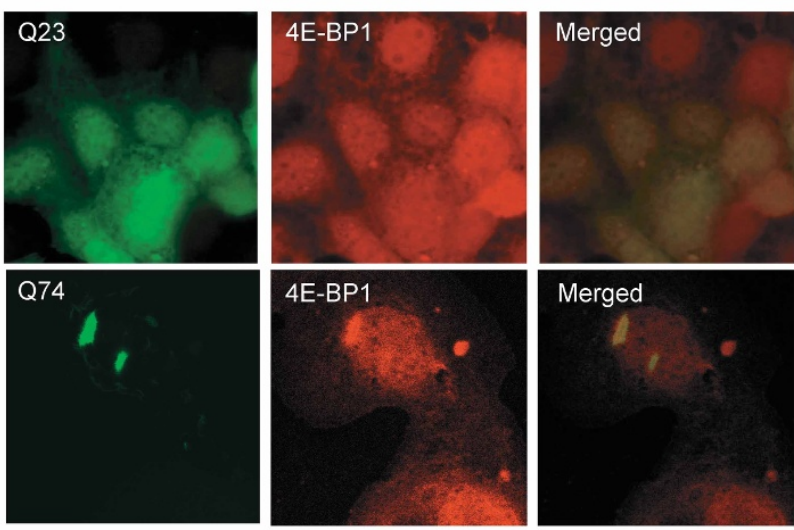

4E-BP1

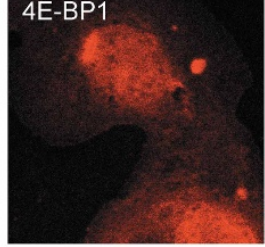

Merged

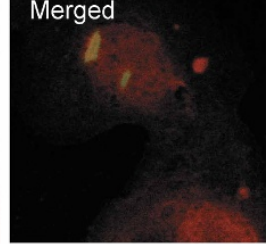

d
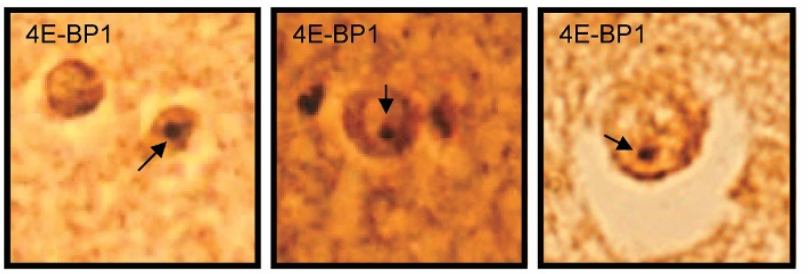

e
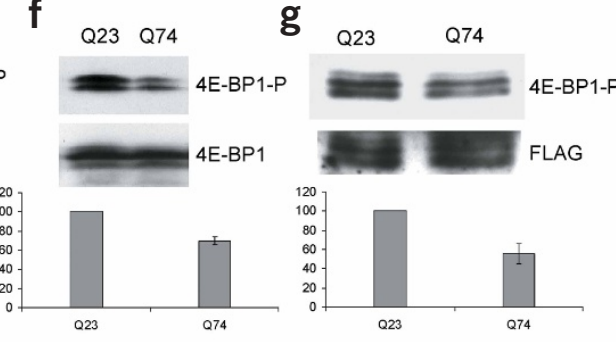
We next tested whether phosphorylation of the ribosomal protein S6, the target of activated, phosphorylated S6K1, was specifically reduced in cells with aggregates, by immunofluorescence ${ }^{21}$. We found that $\sim 26 \%$ ( $\pm 2.6 \%$ s.e.) of mutant cells with aggregates did not show staining for phosphorylated S6, compared with $8.5 \%$ ( $\pm 1.2 \%$ s.e.) and $3.8 \%$ ( $\pm 0.5 \%$ s.e. $)$ of wild-type cells and mutant cells without aggregates, respectively (Fig. 3a). We observed similar trends using huntingtin exon 1 constructs with $25\left(\mathrm{Gln}_{25}\right)$ or $103\left(\mathrm{Gln}_{103}\right)$ glutamine repeats (Supplementary Fig. 3 online) and in brains of mice expressing mutant huntingtin versus control mice, where cells with aggregates had less staining for phosphorylated S6 than cells without aggregates (Fig. 3b). In mouse brains, $\sim 60 \%$ of cells with aggregates had markedly less immunoreactivity to phosphorylated S6, whereas brains of control mice had no obvious reductions ( $<2 \%$ of cells).

Polyglutamine expansion impairs mTOR-dependent translation Phosphorylation of S6 by S6K1 positively regulates the initiation of translation of mRNAs containing $5^{\prime}$ TOPs, such as ribosomal proteins and translation elongation factors. We used a validated luciferase reporter assay to measure $\mathrm{mTOR}$-dependent TOP translation ${ }^{18,22}$ in the presence or absence of rapamycin in order to assess the amount of functional cellular mTOR that can be inhibited. Rapamycin significantly reduced TOP-dependent (+TOP) luciferase expression in wild-type $\left(\mathrm{Gln}_{23}\right)$ PC12 cells after serum stimulation by $\sim 18 \%$ (Fig. $3 \mathrm{c}$ ), similar to results of previous studies with this assay in normal cells ${ }^{18,22}$. This effect is modest because mTOR only partially controls this process ${ }^{18}$. Rapamycin had no such inhibitory effect in mutant $\left(\mathrm{Gln}_{74}\right)$ cells. As expected, rapamycin did not inhibit luciferase activity in wild-type cells transfected with an otherwise identical control-luciferase construct without the TOP sequence (-TOP; Fig. 3c). These data suggest that rapamycin cannot reduce $\mathrm{mTOR}$ activity in mutant cells because it is already impaired, consistent with the raw luciferase data with the +TOP constructs showing lower values in mutant cells in the absence of rapamycin than in wild-type cells (Supplementary Fig. 4 online). The increase in TOP-independent translation in the mutant lines (Fig. 3c) is compatible with data from previous assays ${ }^{18,22}$, in which it was attributed to competition between different classes of mRNA for the cellular translation machinery ${ }^{18}$. But the key issue is the TOP-dependent translation. We confirmed the TOP-dependent effects (Fig. 3c) in COS-7 cells transiently transfected with wild-type or mutant huntingtin along with a
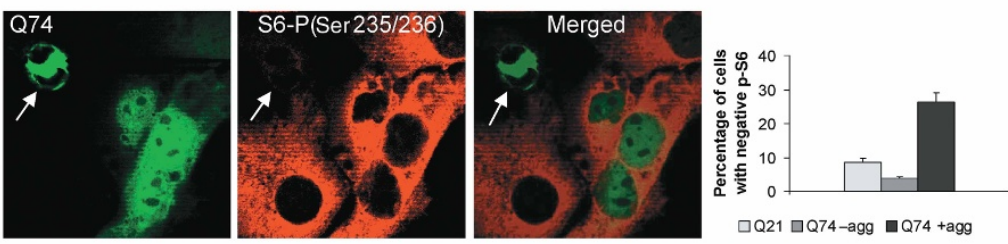

b
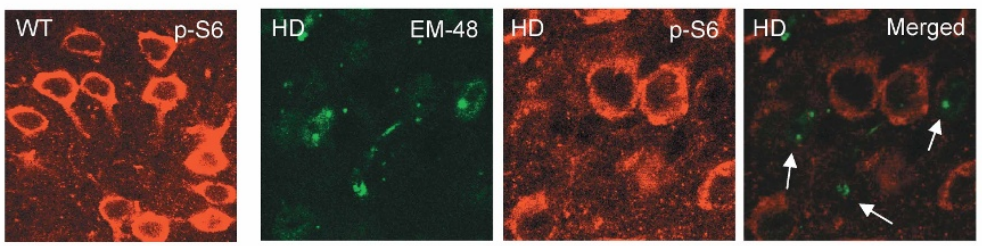

C
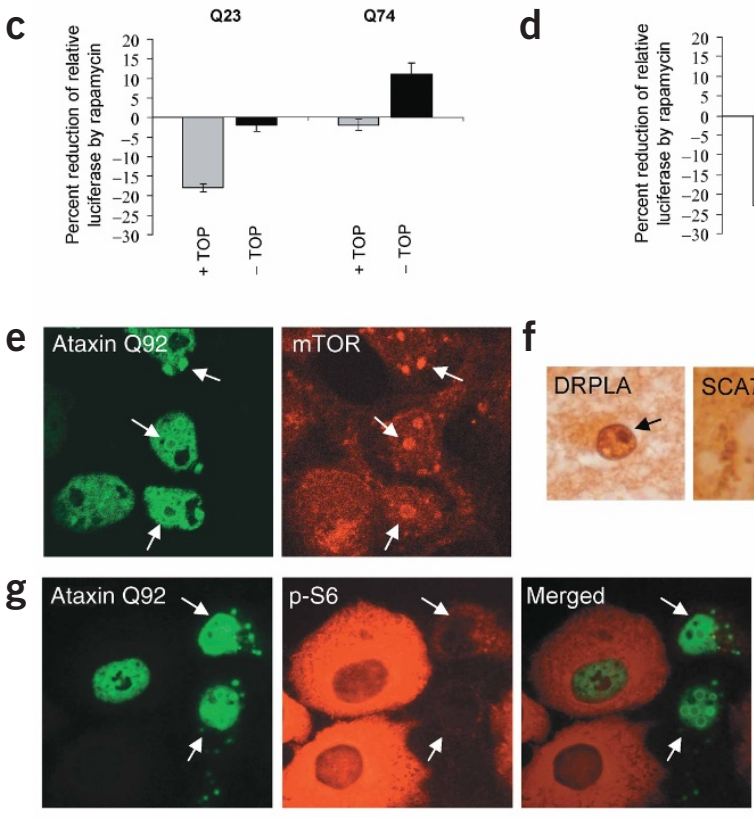

d

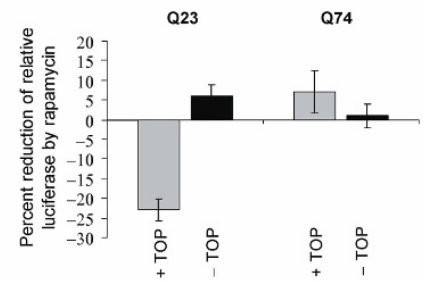

\section{f}
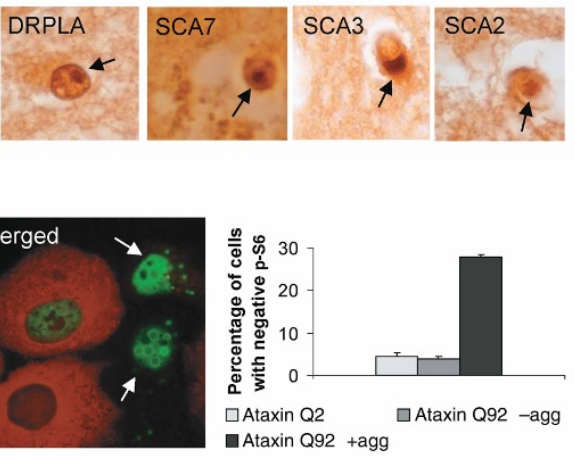

Figure 3 Impaired phosphorylation of ribosomal protein S6 and dysregulation of TOP-dependent translation. (a) COS-7 cells expressing wild-type $\left(\mathrm{Gln}_{23} ; \mathrm{Q} 23\right)$ or mutant $\left(\mathrm{Gln} \mathrm{n}_{74} ; \mathrm{Q74}\right)$ huntingtin (green) were labeled for S6 phosphorylated at Ser235 and Ser236 (red). Percentages of cells expressing wild-type or mutant huntingtin that did (+agg) or did not (-agg) contain aggregates were scored for negative staining for phosphorylated S6 (p-S6). (b) Brain sections from control (WT) and mice expressing mutant huntingtin ${ }^{16}$ (HD) were stained for phosphorylated S6 (red) and with antibody to huntingtin (EM48; green).

(c,d) Impaired mTOR-dependent TOP translation in cells expressing mutant huntingtin. (c) PC12 cells expressing wild-type $\left(\mathrm{Gln}_{23} ; \mathrm{Q} 23\right)$ or mutant $\left(\mathrm{Gln}_{74} ;\right.$ Q74) huntingtin were transfected with luciferase constructs containing $5^{\prime}$ untranslated sequence with ${ }^{22}(+)$ or without (-) a TOP sequence along with $\beta$-gal. Each bar represents the difference between the luciferase activities in the presence versus the absence of rapamycin. Data shown are from one representative experiment (of three) done in sextuplicate (error bars represent s.e.; raw data are given in Supplementary Fig. 4 online). (d) COS-7 cells were cotransfected with wild-type or mutant huntingtin, a nontypical TOP or control luciferase vector ${ }^{18}$ and $\beta$-gal. The experiment was done and analyzed as in c (raw data are given in Supplementary Fig. 4 online) $(\mathbf{e}-\mathbf{g})$ Relevance of mTOR sequestration to other polyglutamine expansion diseases (e) COS-7 cells expressing Xpress-tagged ataxin-1 with 92 polyglutamine repeats (Q92) for $48 \mathrm{~h}$ were doubly labeled with antibodies to Xpress (green) and to mTOR (red). (f) mTOR immunohistochemistry in brains of individuals with dentatorubralpallidoluysian atrophy (DRPLA) and spinocerebellar ataxia types 2, 3 and 7 (SCA2, SCA3 and SCA7). (g) Staining for phosphorylated S6 (red) in COS-7 cells transiently transfected with mutant (ataxin Q92; green) or wild-type (ataxin Q2) ataxin-1 constructs. Results were significant and similar to those in $\mathbf{a}$. Arrows in $\mathbf{a}, \mathbf{b}, \mathbf{e}$ and $\mathbf{g}$ show cells containing aggregates. 
another set of luciferase reporter constructs with a nontypical TOP sequence, whose translation is also normally inhibited by rapamycin treatment ${ }^{18}$ (Fig. 3d). Cells transfected with wild-type huntingtin showed a rapamycin-dependent reduction $(23 \%)$ in luciferase activity that was similar to that previously described ${ }^{18}$, but we observed no such effect in cells transfected with mutant huntingtin, which behaved like cells expressing nonfunctional mTOR mutants ${ }^{18}$. Again, we noted that the raw luciferase data (Fig. $3 \mathbf{d}$ ) with the + TOP constructs showed lower values in mutant cells in the absence of rapamycin than in wild-type cells (Supplementary Fig. 4 online). These data suggest that mutant huntingtin causes translational dysregulation by impairing mTOR signaling to its downstream effectors.

\section{Decreased $\mathrm{mTOR}$ activity in other polyglutamine diseases}

The huntingtin exon 1 constructs that we used previously formed mostly cytoplasmic aggregates (90\% of aggregates in mutant $\mathrm{Gln}_{74}$ cells and $98 \%$ of aggregates in mutant $\operatorname{Gln}_{103}$ cells). But mTOR was also sequestered by intranuclear ataxin-1 aggregates (Fig. 3e), aggregates formed by isolated polyglutamine stretches fused to GFP $\left(\mathrm{Gln}_{81}\right.$; Supplementary Fig. 5 online) and aggregates in brains of individuals with spinocerebellar ataxia types 2, 3 and 7 and dentatorubral-pallidoluysian atrophy (Fig. 3f). We also observed less staining of phosphorylated S6 staining in aggregate-containing COS-7 cells expressing ataxin-1 (Fig. 3g) and mutant $\mathrm{Gln}_{81}$ huntingtin (Supplementary Fig. 5 online), suggesting that mTOR sequestration and inhibition might be polyglutamine-dependent irrespective of location.

\section{Polyglutamine expansion induces autophagy}

mTOR inhibition induces autophagy, which has been previously reported in brains of individuals with Huntington disease, in transgenic mice expressing mutant huntingtin fragments and in a cell model of Huntington disease, using morphological criteria $^{23,24}$. It has been difficult to differentiate autophagosomes from other vesicles in the absence of specific autophagosome markers. This issue is simplified by the identification of LC3-II, a form of microtubule-associated protein-1 light chain-3 (MAP-LC3) associated with mammalian autophagosomes ${ }^{25}$. Levels of LC3-II (relative to actin), which correlate with autophagosome number ${ }^{25}$, were higher in mutant $\left(G \ln _{74}\right)$ than in wild-type $\left(\mathrm{Gln}_{23}\right)$ cell lines (Fig. 4a). Although this effect was subtle, it was consistent and reproducible in four independent experiments. Furthermore, levels of LC3-II increased after expression of mutant huntingtin was induced in stable PC12 cells (data not shown), whereas no difference was seen upon induction of expression of wild-type huntingtin. Autophagic activity also correlates with the number of LC3-stained vesicles ${ }^{25}$, which increased after serum starvation or treatment with rapamycin (known to stimulate autophagy) and in aggregate-containing cells expressing mutant $G \ln _{74}$ or $\mathrm{Gln}_{103}$ huntingtin but not in cells expressing wild-type $\mathrm{Gln}_{23}$ or $\mathrm{Gln}_{25}$ huntingtin (Fig. $\mathbf{4 b}$ and Supplementary Fig. 6 online). The small size of PC12 cells made visualization of LC3-stained vesicles indistinct both in control cells and in cells treated with rapamycin.

The association of mTOR with polyglutamine aggregates and the impairment of mTOR signaling are not due to cell death. We observed these phenomena in mutant PC12 cells at times where cell death was negligible ${ }^{20}$, and cell death was not obvious in the mice expressing mutant huntingtin that we studied ${ }^{16}$. In addition, we observed sequestration of mTOR to mutant huntingtin aggregates in association with decreased S6 phosphorylation and increased autophagy in aggregatecontaining cells with nonapoptotic nuclear morphologies and in cells treated with the pan-caspase inhibitor z-VAD-fmk (Supplementary Figs. 7-9 online).

\section{Increasing mTOR activity enhances polyglutamine toxicity}

Our hypothesis that inhibition of mTOR protects cells with polyglutamine expansion predicts that activation of mTOR would enhance aggregation and toxicity of mutant huntingtin. This cannot be investigated in wild-type cells by overexpressing a

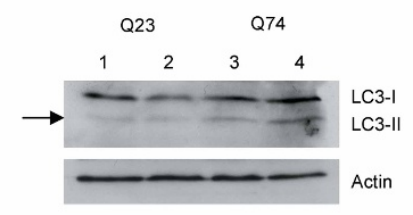

b
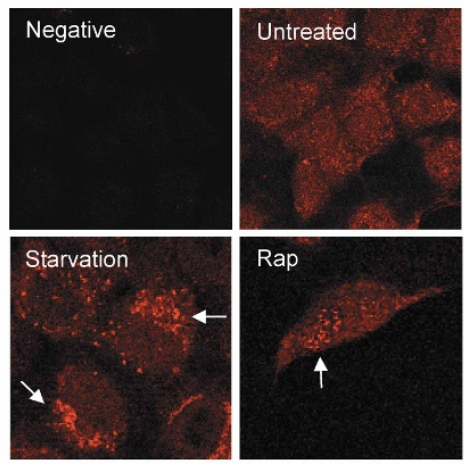

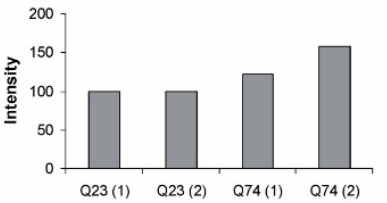

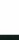
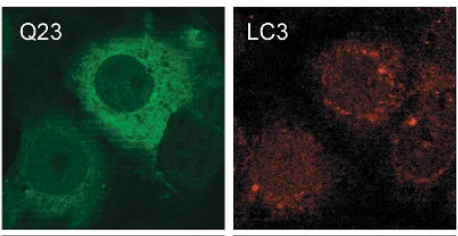
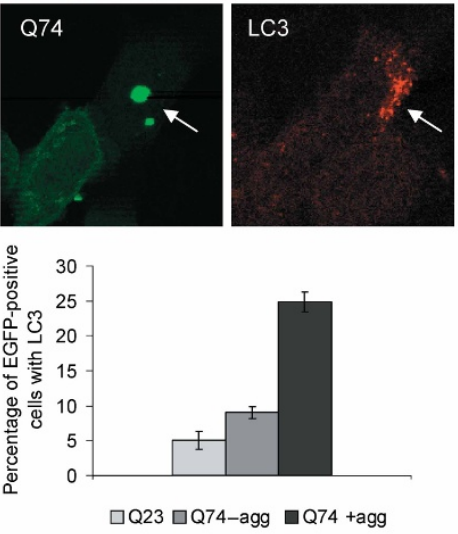

Figure 4 Polyglutamine expansion induces autophagy. (a) LC3-II levels, which closely correlate with autophagic activity, were higher in mutant lines (expressing $\mathrm{Gln}_{74}$ huntingtin; Q74; lanes 3 and 4) than in wild-type lines (expressing Gln $_{23}$ huntingtin; Q23; lanes 1 and 2). Actin was used as a loading control. Data are shown for two independent clonal lines expressing mutant or wild-type huntingtin. Quantification of the band intensity is shown in the right panel. (b) Immunofluorescence analysis of COS-7 cells expressing wild-type $\left(\mathrm{Gln} \mathrm{n}_{23} ; \mathrm{Q} 23\right)$ or mutant $\left(\mathrm{Gl} \mathrm{n}_{74} ; \mathrm{Q74}\right)$ huntingtin with antibody to LC3 showed more autophagic vacuoles in cells expressing mutant huntingtin that had aggregates (arrow). COS-7 cells without the primary antibody (negative) and COS-7 cells without any treatment (untreated) stained for LC3 are also shown. The numbers of EGFP-positive cells expressing wild-type or mutant huntingtin with (+agg) and without (-agg) aggregates that had higher numbers of autophagic vacuoles (>15-20 vesicles per cell) is shown in the graph. COS-7 cells grown in starvation medium (starvation) or treated with rapamycin (Rap) for $4 \mathrm{~h}$ to induce autophagy had more autophagic vacuoles (arrows). 
mTOR, because mTOR overexpression in flies results in phenotypes similar to those caused by mTOR deficiency ${ }^{26}$. Overexpression of the small $G$ protein rheb, which greatly enhances mTOR signaling ${ }^{27}$, however, markedly increased both aggregate formation and cell death caused by mutant huntingtin (Fig. 5a and Supplementary Fig. 10 online).

\section{Rapamycin reduces neurodegeneration in a fly model}

We first tested the therapeutic potential of rapamycin in vivo using fruit flies expressing the first 171 residues of huntingtin with 120 glutamines $\left(\mathrm{Gln}_{120}\right)$ in photoreceptors ${ }^{28}$. The fly's compound eye comprises many ommatidia, each containing eight photoreceptor neurons with light-gathering parts called rhabdomeres, seven of which can be visualized using the pseudopupil technique ${ }^{29}$. The number of visible rhabdomeres in each ommatidium decreases over time in flies expressing mutant $\mathrm{Gln}_{120}$ huntingtin but not in transgenic flies expressing wild-type $\left(\mathrm{Gln}_{23}\right)$ huntingtin ${ }^{28}$. Flies expressing mutant huntingtin that were treated with rapamycin (which was previously shown to effectively inhibit TOR function in flies ${ }^{30}$ ) experienced markedly slower neurodegeneration than those treated with the carrier dimethylsulfoxide (DMSO; used as a control; Fig. 5b,c and Supplementary Fig. 11 online).

\section{$\mathrm{CCl}-779$ relieves symptoms in a mouse model}

Rapamycin has poor water solubility and stability in aqueous solution. The rapamycin ester CCI-779 (ref. 31) has more favorable pharmaceutical properties, induces only mild side effects in humans and is undergoing evaluation in phase 2 and phase 3 clinical trials for treatment of cancer ${ }^{32}$. We confirmed that CCI-779 enhanced clearance of mutant huntingtin exon 1 fragments in a cell model (Supplementary Fig. 12 online) and then used it to treat mice expressing mutant huntingtin. We used Ross/Borchelt mice expressing mutant huntingtin ${ }^{16}$ instead of the more commonly used R6/2 model $^{33}$ for the following reasons. First, rapamycin may be most potent when used before disease onset ${ }^{8}$. R6/2 mice develop pathological signs at 3.5 weeks (ref. 34). Because we intended to start our trial after weaning the mice (around 4 weeks), the later onset in the Ross/Borchelt model made it more suitable. Second, the Ross/Borchelt transgene driven by the mouse prion protein promoter has almost exclusively neuronal expression ${ }^{16}$. R6/2 mice develop inclusions in many peripheral tissues and have muscle atrophy ${ }^{35}$; it is not clear how these peripheral pathologies affect performance on motor-dependent tasks, such as rotarod tests, that are routinely used to assess treatment responses. If the treatment compounds improve muscle (or other peripheral) pathologies in the R6/2 mice but do not cross the blood-brain barrier or act in neurons, these tests could yield misleading positive results.

We identified four tasks or phenotypes that reliably discriminate between the Ross/ Borchelt mice and their wild-type littermates: rotarod test, grip strength test, wire

Figure 5 mTOR activity regulates polyglutamine toxicity in cells and flies. (a) Activation of mTOR activity with rheb increases aggregation and toxicity of mutant $\left(\mathrm{Gln}_{74} ; \mathrm{Q74}\right)$ huntingtin. COS-7 cells were cotransfected with a mammalian expression vector encoding rheb (or empty vector control) and mutant huntingtin for $48 \mathrm{~h}$ at a 3:1 mass ratio (rheb or empty vector to huntingtin) to ensure that almost all cells expressing huntingtin also expressed rheb. The percentages of EGFP-positive cells with aggregates or apoptotic nuclear morphology (as described previously ${ }^{15}$ ) were markedly higher when rheb was overexpressed. Control cells express mutant huntingtin (green) without rheb. The middle panels show images captured at the same comparatively low gain to distinguish aggregates from soluble EGFP. The left panels show the same fields at a similar higher gain to indicate the total number of EGFP-positive cells (this shows that the effect seen in the middle panels is not due to loss of cells). Nuclear staining with DAPI (blue) is shown in the right panels. $* * * P<0.0001$. Error bars represent s.e. for a representative triplicate experiment.

$(\mathbf{b}, \mathbf{c})$ Treatment with rapamycin rescues huntingtininduced degeneration in flies $2 \mathrm{~d}$ after eclosion. Photographs of ommatidia of flies expressing mutant ( $\mathrm{Gl} \mathrm{n}_{120}$; Q120) huntingtin28 treated with DMSO or rapamycin from the larval stage into adulthood. More rhabdomeres are visible in the ommatidia of flies treated with rapamycin. This effect is significant $(P<0.0001$; Mann-Whitney $U$ test). At $2 \mathrm{~d}$ after eclosion, there is no reduction in the number of visible rhabdomeres in wild-type untreated flies (data not shown). 
maneuver test and tremors ${ }^{36}$. Ross/Borchelt mice treated with CCI779 performed significantly better on each of these tasks than their Ross/Borchelt littermates treated with a placebo (Fig. 6 and Supplementary Fig. 13 online). Although weight loss is associated with disease in Ross/Borchelt mice, weight is not a meaningful measure of response to treatment with rapamycin or CCI-779, because CCI-779 reduces weight gain in wild-type mice (Fig. 7a), compatible with previous observations in rodents ${ }^{37}$. These effects may be more severe in mice up to 5 months of age when they are normally gaining weight, as weight loss is not a prominent complication of treatment with rapamycin or CCI-779 in adult humans ${ }^{38}$. Brain weight was reduced in Ross/Borchelt mice treated with CCI-779 compared with Ross/Borchelt mice treated with a placebo (Fig. 7b), but brain weight corrected for body weight was not reduced by this treatment and tended to be higher in the group treated with CCI-779 (Fig. 7c).

There were fewer aggregates in the striatum of mice treated with CCI-779 that in that of mice treated with a placebo. Furthermore, the aggregates of mice treated with CCI-779 were smaller and more difficult to see than those of mice treated with a placebo (Fig. 8a). The difference in aggregate density induced by CCI-779 is probably greater than it appears, as the overall brain mass (and therefore volume) of mice treated with CCI-779 was significantly lower than that of control mice treated with a placebo. These changes are compatible with the idea that CCI-779 impairs mTOR activity and induces autophagy. We confirmed that CCI-779 reduced mTOR signaling in the brains of treated mice, using an antibody specific for phosphorylated S6, as described previously ${ }^{14,21}$ (Fig. 8b).

\section{DISCUSSION}

Our data show that mutant huntingtin interacts with mTOR, which it sequesters in inclusions, leading to decreased mTOR activity. In cells with mutant huntingtin aggregates, mTOR activity was not further reduced by rapamycin treatment. This may explain why rapamycin did not efficiently reduce huntingtin aggregate levels (by autophagy of mutant huntingtin fragments) in populations in which $\sim 30 \%$ of cells contained aggregates but did reduce aggregates in populations in which $10 \%$ of cells contained aggregates ${ }^{8}$.

The lower activity of mTOR in mutant cells is probably due to its tight sequestration in aggregates, leading to reduced soluble mTOR. mTOR may be sequestered by aggregates that are large and visible or smaller and not visible by light microscopy, as mTOR associates with huntingtin that enters stacking or resolving gels. Because there was more total (soluble plus aggregated) mTOR in mutant cells than in wild-type cells (Fig. 1d) and we observed decreased mTOR function in

\begin{abstract}
Figure $6 \mathrm{CCl}-779$ improves behavior and motor performance in a mouse model of Huntington disease ${ }^{16}$. Open bars, mice treated with a placebo; filled bars, mice treated with $\mathrm{CCl}$-779. (a-c) Behavioral tests in mice expressing mutant huntingtin ${ }^{16}$ after treatment at 16 weeks (CCl-779, $n=14$; placebo, $n=14)$, 18 weeks (CCl-779, $n=10$; placebo, $n=9$ ), 20 weeks (CCI-779, $n=9$; placebo, $n=7)$ and 22 weeks (CCl-779, $n=$ 5; placebo, $n=6$ ) of age. Scores are explained in Methods. Data for all time points are given in Supplementary Figure 13 online. (a) Tremors. Overall effect from all treated time points: $P=0.0006$, odds ratio $(\mathrm{OR})=0.21,95 \%$ confidence interval (95\% c.i.) $=0.098-0.45$. 16 weeks, $P=0.01 ; 18$ weeks, $P=0.08 ; 20$ weeks, $P=0.14 ; 22$ weeks, $P=0.07$. (b) Wire maneuver. Overall effect from all treated time points: $P=0.02, \mathrm{OR}=0.51,95 \% \mathrm{c} . \mathrm{i} .=$ 0.29-0.92. 16 weeks, $P=0.29 ; 18$ weeks, $P=0.40 ; 20$ weeks, $P=0.003 ; 22$ weeks, $P=0.008$. (c) Grip strength. Overall effect from all treated time points: $P<0.0001$ $\mathrm{OR}=12.73,95 \%$ c.i. $=4.35-37.2 .16$ weeks, $P=0.1827 ; 18$ weeks, $P=0.0412$; 20 weeks, $P=0.0021 ; 22$ weeks,

$P=0.0285$. (d) Accelerating rotarod tests in mice expressing mutant huntingtin ${ }^{16}$ after treatment at 4 weeks, $(\mathrm{CCl}-779, n=16$; placebo, $n=17)$, 14 weeks (CCl-779, $n=11$; placebo, $n=10), 16$ weeks $(\mathrm{CCl}-779, n=14$; placebo, $n=14)$ and 18 weeks (CCI-779, $n=10$; placebo, $n=9$ ) of age. Overall effect from all treated time points: $P=0.035$. 4 weeks, $P=0.227 ; 14$ weeks, $P=0.012$; 16 weeks, $P=0.0025 ; 18$ weeks, $P=0.057$. $\mathrm{CCl}-779$ had no discernable effects on the performance of nontransgenic mice on any of these behavioral tests (data not shown). There was no difference in the male:female ratios of the groups treated with placebo or with CCl-779.
\end{abstract}

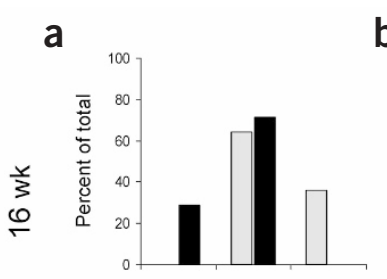

b
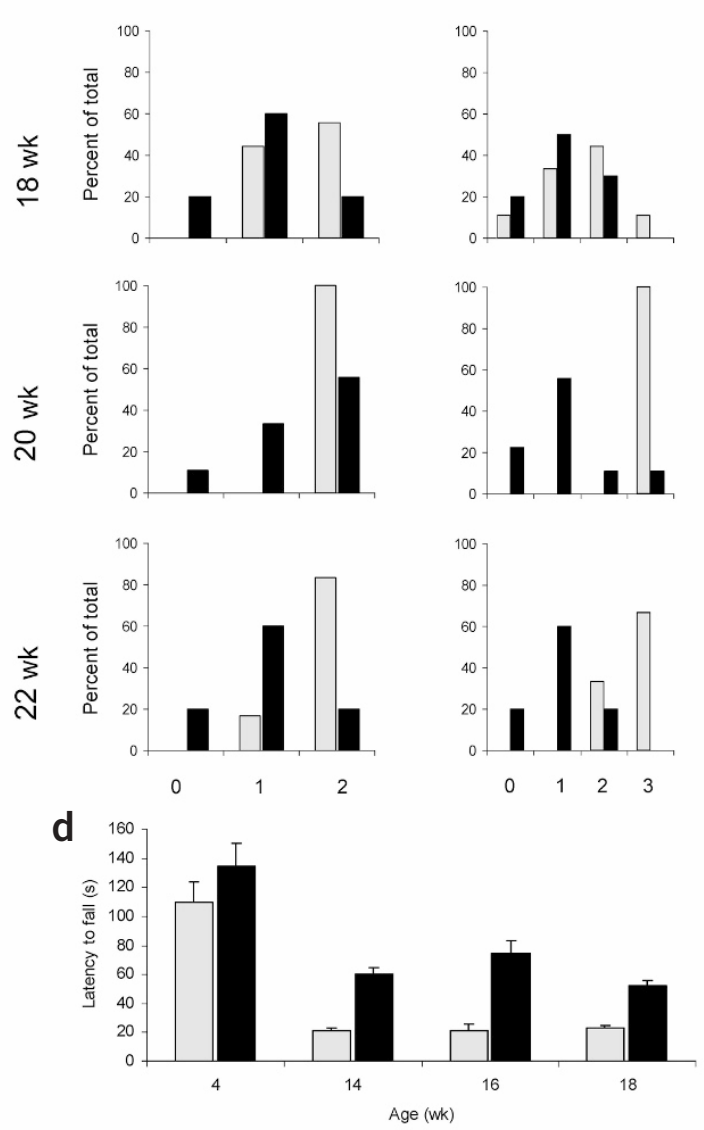

C
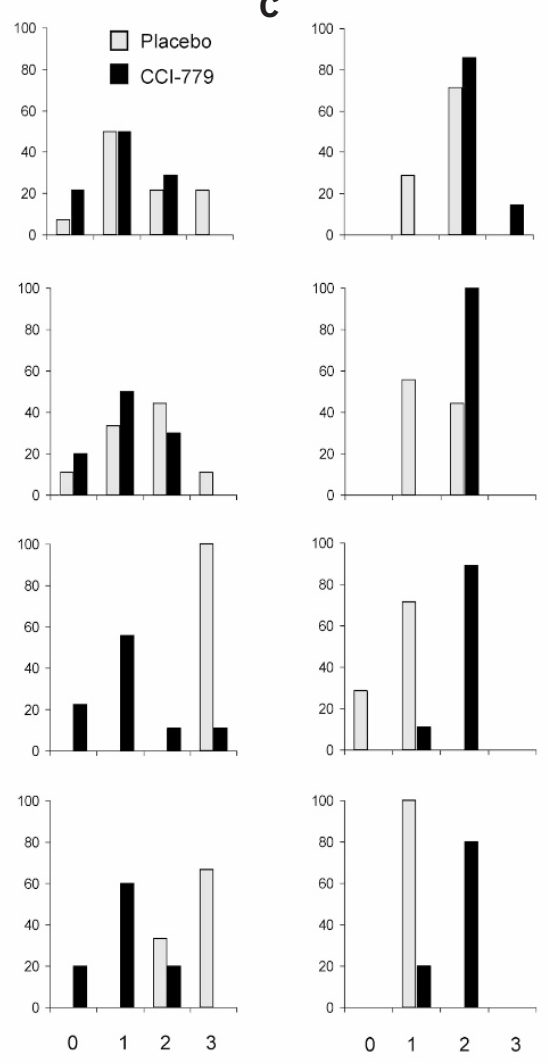
mutant cells, the aggregated mTOR is probably inactivated. This is consistent with nuclear-cytoplasmic shuttling being required for mTOR activity ${ }^{18}$ and is sufficient to explain the decreased activity in cells with polyglutamine expansion. The greater total amounts of mTOR are consistent with the predicted slower turnover of aggregated versus soluble protein.

The overall effect of aggregates may be toxic ${ }^{1}$, but inclusions probably modulate numerous pathways, some toxic and some that reduce polyglutamine toxicity. Impaired mTOR signaling that induces autophagy provides the first molecular basis for a protective pathway induced by polyglutamine aggregates. This may contribute to the typical late onset of Huntington disease and help account for the many surviving cells with aggregates in brains of individuals with Huntington disease $e^{4,39}$. Despite the induction of autophagy, aggregates will accumulate if their production exceeds their clearance. Although aggregates visible by light microscopy are probably too large to be cleared by autophagosomes (typically up to $1.5 \mu \mathrm{m}$ in diameter), microaggregates and soluble huntingtin are compatible in size with these vesicles ${ }^{40}$.

Chronically impaired mTOR activity may also explain neuronal shrinkage, which contributes to much of the brain atrophy seen in Huntington disease ${ }^{1}$. The mTOR target S6K1, which has reduced phosphorylation and activity in cells with mutant huntingtin, is a key regulator of cell volume ${ }^{10}$.

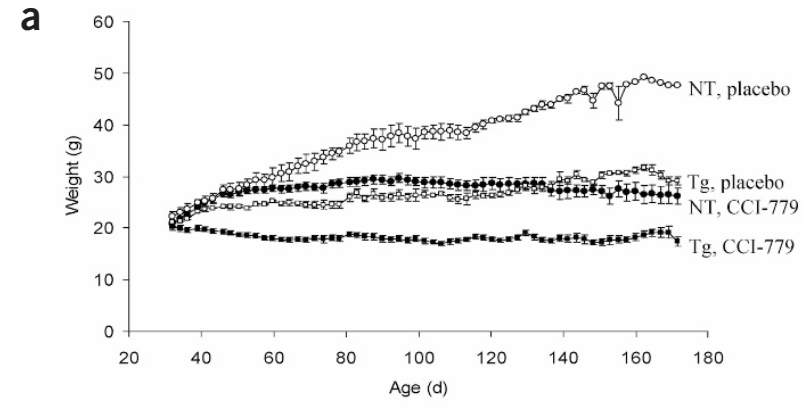

b

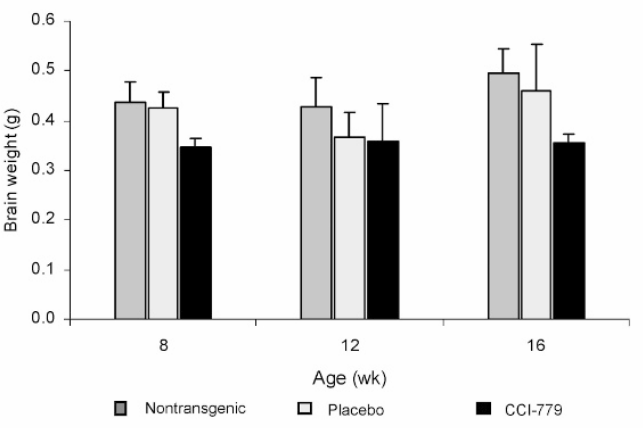

C

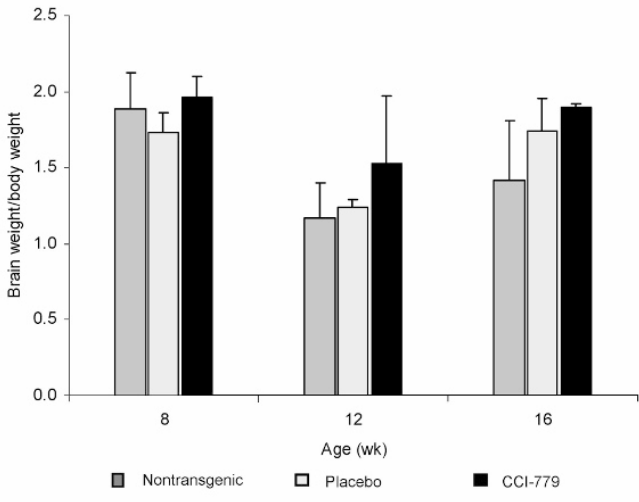

Our data suggest that mTOR inhibition by mutant polyglutamine expansions also affects translation regulated by S6K1 and 4E-BP1 (ref. 43). Because the dependence of gene translation on mTOR activity depends on the sequence and structure of the $5^{\prime}$ untranslated region, this effect will vary from gene to gene ${ }^{41}$. This effect might theoretically contribute to some of the memory defects in Huntington disease and to the defects in long-term potentiation seen in mouse models of polyglutamine disease ${ }^{42-44}$, because long-term potentiation requires mTOR-dependent protein synthesis at synapses ${ }^{45}$. But we are not aware of memory or cognitive problems developing in humans using rapamycin or its analogs in the long term.

Our trials with rapamycin and CCI-779 in fly and mouse models of Huntington disease suggest that the therapeutic potential of mTOR inhibition deserves serious consideration. Rapamycin seems to be more protective than other compounds tested in flies (e.g., $\mathrm{SAHA}^{46}$ ). Mice treated with rapamycin experience improvements in four different phenotypes attributable to neurological dysfunction. We did not observe a significant improvement in lifespan (Supplementary Fig. 14 online), but lifespan measurements were confounded by several factors. First, our license to carry out experiments on mice requires that we euthanize mice when their disease exceeds defined, moderately severe, humane end points. Because $\sim 50 \%$ of mice in each group required euthanasia and because the mice that died spontaneously frequently died without warning, obvious reason or correlation to apparent disease severity ${ }^{16}$, the death data we obtained was not as precise as it would have been if all mice were allowed to die naturally. This circumstance precluded detection of small effects on lifespan. Second, Ross/Borchelt mice have more variable lifespans than R6/2 mice ${ }^{47}$. Third, the cause of death in the Ross/Borchelt mice is unknown and may be more dependent on weight loss than any true Huntington disease-related neurological pathology. Rapamycin markedly reduces weight gain in both transgenic mice and nontransgenic littermates, which may counterbalance the effects of reducing mutant huntingtin levels in the transgenic mice. Because weight loss is not a common complication of rapamycin therapy in adult humans, however, this effect may differ between adult humans and developing mice. Accordingly, we do not believe that the absence of a definite effect on lifespan reduces the potential therapeutic value of rapamycin or CCI-779. Other researchers also do not use lifespan as an indicator in therapeutic trials in mice, which they euthanize at humane end points ${ }^{48}$.

Theoretically, the rapamycin or CCI-779 strategy has some advantages over approaches that aim to attenuate Huntington disease by

Figure $7 \mathrm{CCl}-779$ reduces weight gain in mice. (a) Weights of mice expressing mutant huntingtin (Tg) and nontransgenic (NT) littermates treated with $\mathrm{CCl}-779$ or with a placebo. (b) Brain weights of mice expressing mutant huntingtin treated with $\mathrm{CCl}-779$ (black bars) or with a placebo (white bars) and nontransgenic untreated littermates (gray bars) at 8 weeks (CCl-779, $n=3$; placebo, $n=3$; nontransgenic, $n=7$; $\mathrm{CCl}-779$ versus placebo, $P=0.023$ ), 12 weeks (CCl-779, $n=2$; placebo, $n=3$; nontransgenic, $n=5 ; \mathrm{CCl}-779$ versus placebo, $P=0.90$ ) and 16 weeks (CCl-779, $n=2$; placebo, $n=3$; nontransgenic, $n=2$; $\mathrm{CCl}-779$ versus placebo, $P=0.22$ ) of age. (c) Ratio of brain weight to body weight of mice expressing mutant huntingtin treated with $\mathrm{CCl}-779$ (black bars) or with a placebo (white bars) and nontransgenic untreated littermates (gray bars) at 8 weeks (CCI-779, $n=3$; placebo, $n=3$; nontransgenic, $n=7$; CCI-779 versus placebo, $P=0.06$ ), 12 weeks (CCI-779, $n=2$; placebo, $n=3$; nontransgenic, $n=5$; $\mathrm{CCl}-779$ versus placebo, $P=0.38$ ) and 16 weeks (CCl-779, $n=2$; placebo, $n=3$; nontransgenic, $n=2$; $\mathrm{CCl}-779$ versus placebo, $P=0.56$ ) of age. 
acting on downstream targets such as apoptosis, reactive oxygen species or transcriptional dysregulation (reviewed in ref. 1). Polyglutamine expansion may induce deleterious changes in many parallel and distinct pathways. Therefore, it may be more effective to treat Huntington disease by reducing levels of the toxic mutant protein rather than by rescuing each pathway that is perturbed (particularly as many of these may be unknown). This approach is simplified by the fact that hemizygous loss of function of huntingtin does not cause overt deleterious effects in humans or mice (reviewed in ref. 1). Although rapamycin or CCI-779 may lose efficacy after aggregate pathology is well developed, this does not preclude its use to treat Huntington disease. The median age at onset of Huntington disease is at least 40 years, and almost all cases have family histories. Because polyglutamine expansion status can be assessed before symptoms appear, one possible treatment strategy would be to delay disease onset with therapy initiated at the earliest feasible age. If disease onset could be delayed beyond normal life expectancy, then one would effectively cure many cases. Because CCI-779 and rapamycin are designed for long-term use in humans, they deserve serious consideration; the benefits of delaying onset of Huntington disease would outweigh the side effects of these drugs. In addition to Huntington disease, we believe that treatment with rapamycin or CCI-779 might be effective for other neurodegenerative diseases, as rapamycin enhances the clearance of cytosolic model aggregate-prone proteins with either polyglutamine or polyalanine expansions as well as various forms of $\alpha$-synuclein associated with Parkinson disease and synucleinopathies in cell models ${ }^{8,9}$.

Figure $8 \mathrm{CCl}-779$ reduces mTOR activity and aggregate load in mice expressing mutant huntingtin. (a) $\mathrm{CCl}-779$ reduces aggregate load in mice expressing mutant huntingtin. Peroxidase immunohistochemistry of brains of mice expressing mutant huntingtin at 16 weeks of age. Mice treated with $\mathrm{CCl}-779$ had fewer aggregates per unit area in the striatum than mice treated with a placebo. Overall cell staining in the tissues of mice treated with $\mathrm{CCl}-779$ was weaker than in those treated with a placebo and in nontransgenic untreated littermates (data not shown). Aggregates, when visible, were smaller in mice treated with CCl-779 than in those treated with a placebo, as apparent in the higher magnification images of single cells with aggregate (bottom panels). Six coronal sections per mouse were analyzed; these corresponded to the bregma $\pm 1 \mathrm{~mm}$. We counted aggregates in five different high-power fields (60x objective) per slide on each side of the brain, sampling fields from the dorsal to the ventral part of the striatum just underneath the external capsule. The striata of mice treated with $\mathrm{CCl}-779$ had $70 \%$ fewer aggregates than the striata of mice treated with a placebo $(P=0.0001$, two-tailed $t$ test). (b) $\mathrm{CCl}-779$ blocks mTOR signaling in vivo. Immunohistochemistry of mice with Huntington disease at 16 weeks of age with antibody to phosphorylated S6, in the cortical area and striatum. Levels of phosphorylated S6 were lower in mice treated with $\mathrm{CCl}-779$ than in those treated with a placebo.

\section{METHODS}

Mammalian cell culture and transfection. We grew African green monkey kidney cells (COS-7) in Dulbecco's modified Eagle medium (Sigma) supplemented with $10 \%$ fetal bovine serum, $100 \mathrm{U} \mathrm{ml}^{-1}$ of penicillin/streptomycin, $2 \mathrm{mM}$ L-glutamine and $1 \mathrm{mM}$ sodium pyruvate at $37^{\circ} \mathrm{C}$ in $5 \%$ carbon dioxide. We maintained the PC12 stable cells with $75 \mu \mathrm{g} \mathrm{ml}^{-1}$ of hygromycin in standard medium consisting of high-glucose Dulbecco's modified Eagle medium (Sigma) with $100 \mathrm{U} \mathrm{ml}^{-1}$ of penicillin/streptomycin, $2 \mathrm{mM}$ L-glutamine (Invitrogen), 10\% heat-inactivated horse serum (Invitrogen), 5\% Tet-approved fetal bovine serum (Clontech) and $100 \mu \mathrm{g} \mathrm{ml}^{-1}$ of G418 (Invitrogen) at $37^{\circ} \mathrm{C}$ in $10 \%$ carbon dioxide. We seeded $1-2 \times 10^{5}$ cells per well in six-well plates and induced them with $1 \mu \mathrm{g} \mathrm{ml}^{-1}$ of doxycycline (Sigma). We carried out transient transfection using LipofectAMINE reagent (Invitrogen) using the manufacturer's protocol. CCI-779 was provided by Wyeth Pharmaceuticals. For tissue culture experiments, we prepared a stock solution of $1 \mathrm{M}$ in ethanol on the day of experiment and diluted it in the appropriate medium. We confirmed all data reported in this paper in the stable inducible PC12 cells in two independent sets of wild-type $\left(\mathrm{Gln}_{23}\right)$ and mutant $\left(\mathrm{Gln}_{74}\right)$ clonal lines.

Western-blot analysis. We carried out western-blot analysis using standard techniques with ECL or ECL Plus detection kit (Amersham). Primary antibodies included antibodies to GFP (Clontech), mTOR, phosphorylated mTOR (Ser2448), S6K1, phosphorylated S6K1 (Thr389; 1A5), 4E-BP1 and phosphorylated 4E-BP1 (Thr37) from Cell Signaling Technology; antibody to huntingtin (EM48, Chemicon); and antibody to tubulin (Sigma). We used brains from nine mice expressing mutant huntingtin ( $\mathrm{N}$-terminal 171 amino acids

\section{a}
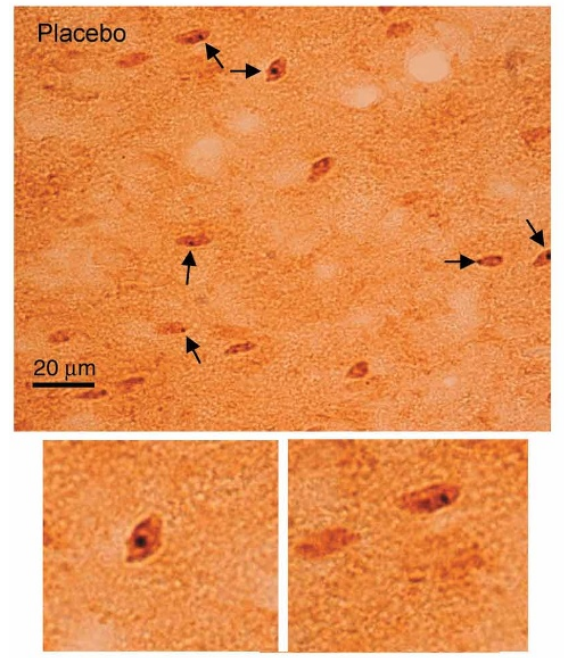

b
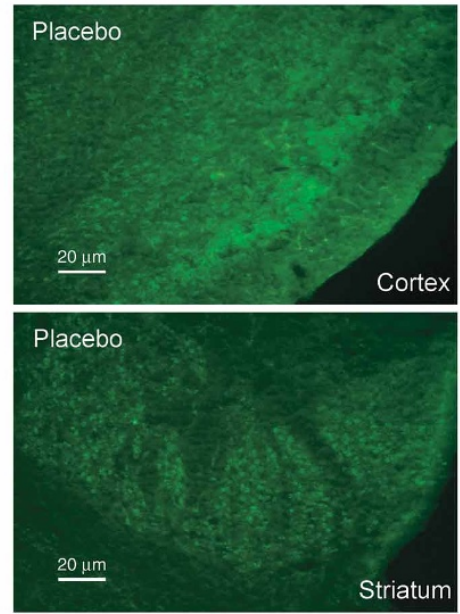
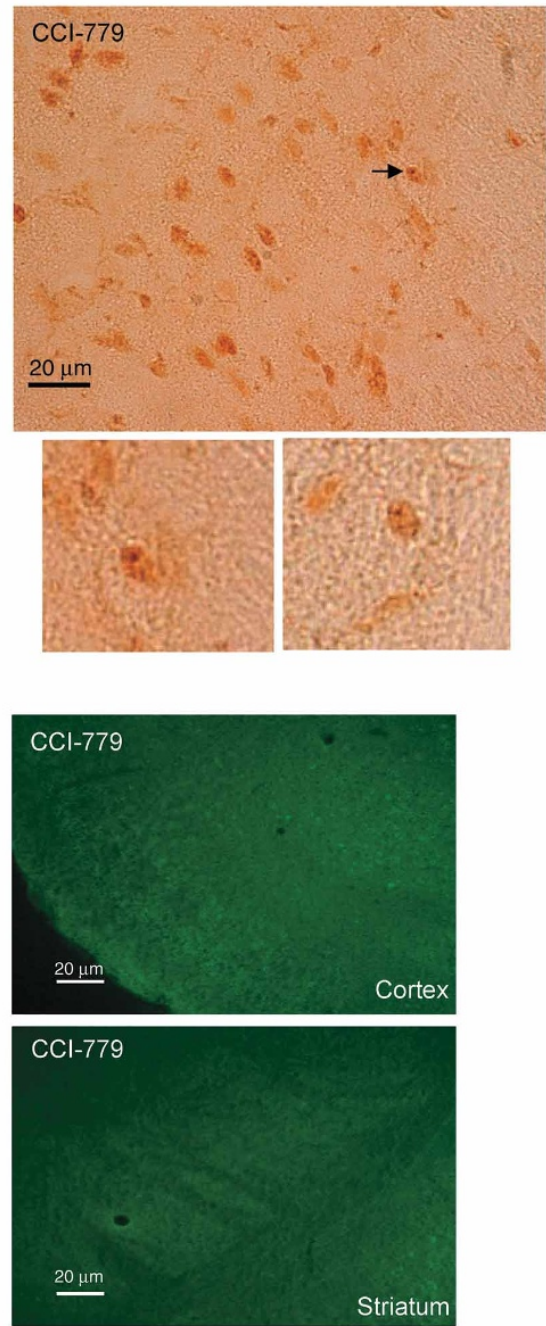
with 82 glutamine repeats) ${ }^{16}$ and age-matched wild-type littermate controls. We added $2.5 \times$ volume of buffer B ( $50 \mathrm{mM}$ Tris ( $\mathrm{pH} 7.5$ ), $10 \%$ glycerol, $5 \mathrm{mM}$ magnesium acetate, $0.2 \mathrm{mM}$ EDTA, $0.5 \mathrm{mM}$ dithiothreitol and protease inhibitor) to sliced brain tissue and homogenized it at $4{ }^{\circ} \mathrm{C}$. We centrifuged the homogenate at 12,000 r.p.m. at $4{ }^{\circ} \mathrm{C}$. We removed the supernatant and used it for western blotting. For immunoprecipitation, we lysed cells in RIPA buffer ( $1 \times$ phosphate-buffered saline, $1 \%$ Nonidet P- $40,0.5 \%$ sodium deoxycholate and $0.1 \%$ SDS) and immunoprecipitated them using relevant antibodies. We carried out densitometry analysis using Scion Image Beta 4.02 software.

Assay of exogenous 4E-BP1 phosphorylation. We transiently transfected PC12 cells stably expressing wild-type $\left(\mathrm{Gln}_{23}\right)$ or mutant $\left(\mathrm{Gln}_{74}\right)$ huntingtin with FLAG-tagged 4E-BP1 for $72 \mathrm{~h}$ and simultaneously induced the transgene. We maintained the cells in normal growth medium for $48 \mathrm{~h}$ and then transferred them to starvation medium for $24 \mathrm{~h}$. At this time point $>99 \%$ of the mutant $\mathrm{PC} 12$ cells formed insoluble mutant aggregates ${ }^{20}$. We then stimulated mTOR activity with $10 \%$ fetal bovine serum for $1 \mathrm{~h}$ and processed the cells for immunoprecipitation. We immunoprecipitated cells with antibody to FLAG and immunoblotted them with both antibody to 4E-BP1 (Thr36/37) and antibody to FLAG.

Immunocytochemistry and immunohistochemistry. We carried out immunocytochemistry in COS-7 cells fixed with 4\% paraformaldehyde (Sigma). For histochemical analysis, we used free-floating brain sections from mice expressing mutant huntingtin ${ }^{16}$ and control mice and paraffin-embedded brain slices from humans with grade III Huntington disease and unaffected humans, spanning the caudate and putamen regions. Primary antibodies included antibodies to mTOR, phosphorylated mTOR (Ser2448), 4E-BP1, phosphorylated S6 (Ser235/236; Cell Signaling Technologies), LC3 (gift from T. Yoshimori, National Institute of Genetics, Japan), huntingtin and ubiquitin (both from Chemicon). We carried out immunohistochemical analysis by standard fluorescence methods or peroxidase labeling using the Vectastain Avidin: Biotinylated enzyme Complex (ABC) kit. We analyzed relevant negative controls without the primary antibodies alongside all experiments.

Luciferase assay. We transfected PC12 cells stably expressing wild-type $\left(\mathrm{Gln}_{23}\right)$ or mutant $\left(\mathrm{Gln}_{74}\right)$ huntingtin with a luciferase construct that contained a 5 untranslated region from the eEF2 gene with or without the TOP sequence along with $\beta$-galactosidase ( $\beta$-gal; to control transfection efficiency) ${ }^{22}$. We maintained transfected cells in serum-free medium with doxycycline for $36 \mathrm{~h}$ and then stimulated the cells with $15 \%$ serum with or without rapamycin for $3 \mathrm{~h}$. We then lysed cells and carried out luciferase assays according to standard protocol. We cotransfected COS-7 cells with wild-type or mutant huntingtin and with the control or TOP luciferase constructs along with $\beta$-gal for $48 \mathrm{~h}$ and carried out experiments as described above. In addition to the classical TOP construct, we also used a construct with a nonclassical TOP sequence that is sensitive to rapamycin and an appropriate control construct ${ }^{18}$. As both control and TOP luciferase constructs have the same promoters and transfection is controlled for with $\beta$-gal construct, the rapamycin-sensitive reduction in luciferase activity that is specific to the TOP construct is considered an indication of the dependency of the translation of the TOP construct of mTOR regulation ${ }^{18,22}$. We carried out experiments in sextuplicate and repeated them several times.

Treatment of flies with rapamycin. We obtained a Drosophila melanogaster $y w ; g m r-Q 120$ line from G. Jackson (University of California Los Angeles, USA) and crossed it to a w[1118] stock isogenized for the X chromosome and two major autosomes, which has been characterized for a number of behaviors and used as the genetic background for the European Drosophila Deletion Kit (DrosDel Consortium). The crossed flies were allowed to mate on normal food for $2-3 \mathrm{~d}$ and then transferred to food containing $1 \mu \mathrm{M}$ rapamycin (Sigma) or DMSO. After eclosion, we placed the flies on food containing $1 \mu \mathrm{M}$ rapamycin or DMSO. We transferred the flies to newly prepared food every day.

Pseudopupil analysis of flies. We removed the heads of adult male flies and then mounted them onto microscope slides using nail polish. We carried out pseudopupil analysis with an optical microscope using a $60 \times$ objective with the observer blinded to the identity of the slides. We evaluated $\sim 75$ ommatidia for each group ( 15 ommatidia from five individuals). Each experiment was done twice. The frequency of ommatidia with different numbers of visible rhabdomeres was based on all visible photoreceptor neurons, irrespective of their shape or brightness. We raised the flies at $25^{\circ} \mathrm{C}$ with 12 -h light:12-h dark cycle in $70 \%$ humidity.

Mice and behavioral tests. All studies and procedures were done under the jurisdiction of appropriate Home Office Project and Personal animal licenses and with local ethical committee approval. We genotyped HD-N171-N82Q mice ${ }^{16}$ expressing the first 171 amino acids of human huntingtin under the expression of a mouse PrP promoter at 3 weeks of age by PCR (primer sequences are available on request). We prepared CCI-779 in ethanol as a stock solution at $50 \mathrm{mg} \mathrm{ml}^{-1}$ on the day of experiment and diluted it to $2 \mathrm{mg} \mathrm{ml}^{-1}$ in $0.15 \mathrm{M} \mathrm{NaCl}, 5 \%$ Tween 20 and 5\% PEG 400 immediately before injection. We compared transgenic mice with nontransgenic littermates. We coded mice with alphanumeric identities that provided no clues to their genotype or treatment status, and treated and untreated mice were not housed in separate cages. Thus, observers were blind to their treatment and genetic status during testing. There were no significant differences in test performances in the mice assigned to the treatment and placebo groups at 4 weeks and no differences in the sex ratios in the groups. We weighed mice three times per week and then injected them intraperitoneally with a $1 \% \mathrm{v} / \mathrm{w}$ solution of CCI-779 (20 mg per $\mathrm{kg}$ body weight) or placebo (equivalent solution without CCI-779). We did three injections every week from the ages of 4-16 weeks, and then did this every other week until they reached 21 weeks of age. We monitored mice daily.

We assessed motor performance at 4, 14, 16 and 18 weeks of age with a rotarod apparatus (Accelerating Model, Ugo Basile, Biological Research Apparatus); our license limited rotarod assessments to four testing time points. The mice were given three training sessions per day for two consecutive days to adapt to the apparatus. On the third day, the mice were given six separate trials. On the training and testing days, we set the speed of the rotarod to increase from 4 r.p.m. to 40 r.p.m. in 250 s; we kept the mice on the rotarod for no more than $300 \mathrm{~s}$. We did not inject mice during the weeks that they were tested on the rotarod to avoid any confounding effects of the injections.

Grip strength, wire maneuver and tremor monitoring are part of the SHIRPA battery of behavioral tests ${ }^{36}$. We assessed performance on these tests in mice at 4, 14, 16, 18, 20 and 22 weeks of age. To assess grip strength, mice were allowed to hold a metal grid with their forelimbs, lifted by the tail so that the hindlimbs were not in contact with the grid and gently pulled backwards by the tail until they could no longer hold the grid. Grip strength was scored as follows: 0 , none; 1 , slight grip, semi-effective; 2 , moderate grip, effective; 3 , active grip, effective. For tremors, the mice were placed on a grid in a clear Perspex cylinder for $5 \mathrm{~min}$. We recorded tremors for the last $2 \mathrm{~min}$ and scored the mice as follows: 0 , none; 1 , mild; 2 , marked. For the wire maneuver, mice were held above a horizontal wire by the tail and lowered to allow the forelimbs to grip the wire. The mice were held in extension, rotated around to the horizontal and released. We scored them as follows: 0 , active grip with hind legs; 1 , difficulty grasping with hind legs; 2 , unable to grasp with hind legs; 3 , unable to lift hind legs, falls within $10 \mathrm{~s}$; 4 , falls immediately.

Statistics. We determined significance levels for comparisons between groups using $t$-tests, repeated-measures or factorial ANOVA, as appropriate, for parametric data and using Mann-Whitney $U$ tests for nonparametric data, using the STATVIEW software, version 4.53 (Abacus Concepts). For mouse behavioral data that were nonparametric and analyzed at multiple time points (tremors, grip strength and wire maneuver), we used the following strategy to provide a measure of the significance of the pooled data across all the treated time points. We compared scores between the treated and control groups using a logistic regression approach. We assessed the effect size in terms of the odds ratio per unit change in score. To allow for the fact that the same mice were observed repeatedly, we assessed significance levels by simulation. To do this, the treatment groups were randomly permuted among the mice 10,000 times, and the significance level was determined as the proportion of simulations for which the $\mathrm{Z}$ score for the treatment effect from the logistic regression exceeded the observed value. We obtained $95 \%$ confidence limits for the odds ratio by using the robust variance approach. We did all calculations in Stata, version 7.0 (StataCorp). We analyzed nonparametric mouse data at individual time points by Mann-Whitney and rotarod by repeated-measures ANOVA. 
Note: Supplementary information is available on the Nature Genetics website.

\section{ACKNOWLEDGMENTS}

We thank Wyeth Pharmaceuticals for the gift of CCI-779; G. Jackson for the fly model of Huntington disease; E. J. L. Maller and J. Chen for control and TOP luciferase vectors; N. Khan for brain samples from humans with Huntington disease and controls; A. Tobin for $\mathrm{Gln}_{25}$ and $\mathrm{Gln}_{103}$ constructs; H. Zoghbi for the ataxin constructs; W. J. Strittmatter for $\mathrm{Gln}_{19}$ and $\mathrm{Gln}_{81}$ constructs; K. L. Guan for rheb and FLAG-4E-BP1 constructs; T. Yoshimori for the antibody to LC3; A. Acevedo and E. Terrenoire for help with mouse brain samples; R. Padinjat for help with pseudopupil illumination; and M. Bobrow, D. Clayton and J. D. Cooper for helpful suggestions. We acknowledge funding from the Commonwealth Scholarship Commission for Ph.D. scholarship (B.R.), the Wellcome Trust for a Senior Clinical Research Fellowship (D.C.R.), a Senior Fellowship in Basic Biomedical Science (R.D.), a Prize Studentship (Z.B.), The Cambridge Overseas Trust (Z.B.), The Biotechnology and Biological Sciences Research Council for a Career Development Award (C.J.O.) and a Medical Research Council programme grant to D.C.R and S. Brown.

\section{COMPETING INTERESTS STATEMENT}

The authors declare that they have no competing financial interests.

Received 16 January; accepted 16 April 2004

Published online at http://www.nature.com/naturegenetics/

1. Rubinsztein, D.C. Lessons from animal models of Huntington's disease. Trends Genet. 18, 202-209 (2002).

2. Davies, S.W. et al. Formation of neuronal intranuclear inclusions underlies the neurological dysfunction in mice transgenic for the HD mutation. Cel/ 90, 537-548 (1997).

3. Saudou, F., Finkbeiner, S., Devys, D. \& Greenberg, M.E. Huntingtin acts in the nucleus to induce apoptosis but death does not correlate with the formation of intranuclear inclusions. Cel/ 95, 55-66 (1998).

4. Gutekunst, C.A. et al. Nuclear and neuropil aggregates in Huntington's disease: relationship to neuropathology. J. Neurosci. 19, 2522-2534 (1999).

5. Dunah, A.W. et al. Sp1 and TAFII130 transcriptional activity disrupted in early Huntington's disease. Science 296, 2238-2243 (2002).

6. La Spada, A.R. \& Taylor, J.P. Polyglutamines placed into context. Neuron 38, 681-684 (2003).

7. Chai, Y., Wu, L., Griffin, J.D. \& Paulson, H.L. The role of protein composition in specifying nuclear inclusion formation in polyglutamine disease. J. Biol. Chem. 276 44889-44897 (2001).

8. Ravikumar, B., Duden, R. \& Rubinsztein, D.C. Aggregate-prone proteins with polyglutamine and polyalanine expansions are degraded by autophagy. Hum. Mol. Genet. 11, 1107-1117 (2002).

9. Webb, J.L., Ravikumar, B., Atkins, J., Skepper, J.N. \& Rubinsztein, D.C. $\alpha$-Synuclein is degraded by both autophagy and the proteasome. J. Biol. Chem. 278, 25009-25013 (2003).

10. Schmelzle, T. \& Hall, M.N. TOR, a central controller of cell growth. Cell 103 253-262 (2000)

11 Huang, S., Bjornsti, M.A. \& Houghton, P.J. Rapamycins: mechanism of action and cellular resistance. Cancer. Biol. Ther. 2, 222-232 (2003).

12. Serkova, N. et al. Sirolimus, but not the structurally related RAD (everolimus), enhances the negative effects of cyclosporine on mitochondrial metabolism in the rat brain. Br. J. Pharmacol. 133, 875-885 (2001).

13. Supko, J.G. \& Malspeis, L. Dose-dependent pharmacokinetics of rapamycin-28-n-ndimethylglycinate in the mouse. Cancer Chemother. Pharmacol. 33, 325-330 (1994).

14. Kwon, C.-H., Zhu, X., Zhang, J. \& Baker, S.J. mTOR is required for hypertrophy of Pten-deficient neuronal soma in vivo. Proc. Natl. Acad. Sci. USA 100, 12923-12928 (2003).

15. Wyttenbach, A. et al. Effects of heat shock, heat shock protein 40 (HDJ-2), and proteasome inhibition on protein aggregation in cellular models of Huntington's disease. Proc. Natl. Acad. Sci. USA 97, 2898-2903 (2000).

16. Schilling, G. et al. Intranuclear inclusions and neuritic aggregates in transgenic mice expressing a mutant N-terminal fragment of huntingtin. Hum. Mol. Genet. 8, 397-407 (1999)

17. Waelter, S. et al. Accumulation of mutant huntingtin fragments in aggresome-like inclusion bodies as a result of insufficient protein degradation. Mol. Biol. Cell 12, 1393-1407 (2001).

18. Kim, J.E. \& Chen, J. Cytoplasmic-nuclear shuttling of FKBP12-rapamycin-associated protein is involved in rapamycin-sensitive signaling and translation initiation. Proc.
Natl. Acad. Sci. USA 97, 14340-14345 (2000).

19. Fingar, D.C., Salama, S., Tsou, C., Harlow, E. \& Blenis, J. Mammalian cell size is controlled by mTOR and its downstream targets S6K1 and 4EBP1/elF4E. Genes Dev. 16 1472-1487 (2002).

20. Wyttenbach, A. et al. Polyglutamine expansions cause decreased CRE-mediated transcription and early gene expression changes prior to cell death in an inducible cell model of Huntington's disease. Hum. Mol. Genet. 10, 1829-1845 (2001).

21. Kim, D.H. et al. GbetaL, a positive regulator of the rapamycin-sensitive pathway required for the nutrient-sensitive interaction between raptor and mTOR. Mol. Cell 11, 895-904 (2003).

22. Schwab, M.S. et al. p70(S6K) controls selective mRNA translation during oocyte maturation and early embryogenesis in Xenopus laevis. Mol. Cell. Biol. 19, 2485-2494 (1999).

23. Kegel, K.B. et al. Huntingtin expression stimulates endosomal-lysosomal activity, endosome tubulation, and autophagy. J. Neurosci. 20, 7268-7278 (2000).

24. Sapp, E. et al. Huntingtin localization in brains of normal and Huntington's disease patients. Ann. Neurol. 42, 604-612 (1997).

25. Kabeya, Y. et al. LC3, a mammalian homologue of yeast Apg8p, is localized in autophagosome membranes after processing. EMBO J. 19, 5720-5728 (2000).

26. Hennig, K.M. \& Neufeld, T.P. Inhibition of cellular growth and proliferation by dTOR overexpression in Drosophila. Genesis 34, 107-110 (2002).

27. Manning, B.D. \& Cantley, L.C. Rheb fills a GAP between TSC and TOR. Trends Biochem. Sci. 28, 573-576 (2003).

28. Jackson, G.R. et al. Polyglutamine-expanded human huntingtin transgenes induce degeneration of Drosophila photoreceptor neurons. Neuron 21, 633-642 (1998).

29. Franceschini, N. \& Kirschfeld, K. Pseudopupil phenomena in the compound eye of drosophila. Kybernetik 9, 159-182 (1971).

30. Zhang, H., Stallock, J.P., Ng, J.C., Reinhard, C. \& Neufeld, T.P. Regulation of cellular growth by the Drosophila target of rapamycin dTOR. Genes Dev. 14, 2712-2724 (2000).

31. Huang, S. \& Houghton, P.J. Mechanisms of resistance to rapamycins. Drug Resist. Update 4, 378-391 (2001).

32. Elit, L. CCI-779 Wyeth. Curr. Opin. Investig. Drugs 3, 1249-1253 (2002).

33. Mangiarini L. et al. Exon 1 of the HD gene with an expanded CAG repeat is sufficient to cause a progressive neurological phenotype in transgenic mice.Ce// 87, 493-506 (1996).

34. Lione, L.A. et al. Selective discrimination learning impairments in mice expressing the human Huntington's disease mutation J. Neurosci. 19, 10428-10437 (1999).

35. Sathasivam, K. et al. Formation of polyglutamine inclusions in non-CNS tissue. Hum Mol. Genet. 8, 813-822 (1999).

36. Rogers, D.C. et al. Behavioral and functional analysis of mouse phenotype: SHIRPA, a proposed protocol for comprehensive phenotype assessment. Mamm. Genome 8, 711-3 (1997).

37. Walpoth, B.H. et al. Prevention of neointimal proliferation by immunosuppression in synthetic vascular grafts. Eur. J. Cardiothorac. Surg. 19, 487-492 (2001).

38. British Medical Association and Royal Pharmaceutical Society of Great Britain. British National Formulary 4 (2003).

39. Kuemmerle, S. et al. Huntington aggregates may not predict neuronal death in Huntington's disease. Ann. Neurol. 46, 842-849 (1999).

40. Mizushima, N., Ohsumi, Y. \& Yoshimori, T. Autophagosome formation in Mammalian cells. Cell Struct. Funct. 27, 421-429 (2002).

41. Gingras, A.C., Raught, B. \& Sonenberg, N. Control of translation by the target of rapamycin proteins. Prog. Mol. Subcell. Biol. 27, 143-174 (2001).

42. Usdin, M.T., Shelbourne, P.F., Myers, R.M. \& Madison, D.V. Impaired synaptic plasticity in mice carrying the Huntington's disease mutation. Hum. Mol. Genet. 8, 839-846 (1999).

43. Murphy, K.P. et al. Abnormal synaptic plasticity and impaired spatial cognition in mice transgenic for exon 1 of the human Huntington's disease mutation. J. Neurosci. 20, 5115-5123 (2000).

44. Yoo, S.Y. et al. SCA7 knockin mice model human SCA7 and reveal gradual accumulation of mutant ataxin-7 in neurons and abnormalities in short-term plasticity. Neuron 37, 383-401 (2003).

45. Tang, S.J. et al. A rapamycin-sensitive signaling pathway contributes to long-term synaptic plasticity in the hippocampus. Proc. Natl. Acad. Sci. USA 99, 467-472 (2002).

46. Steffan, J.S. et al. Histone deacetylase inhibitors arrest polyglutamine-dependent neurodegeneration in Drosophila. Nature 413, 739-743 (2001).

47. Ferrante, R.J. et al. Therapeutic effects of coenzyme Q10 and remacemide in transgenic mouse models of Huntington's Disease. J. Neurosci. 22, 1592-1599 (2002).

48. Hockly, E. et al. Suberoylanilide hydroxamic acid, a histone deacetylase inhibitor, ameliorates motor deficits in a mouse model of Huntington's disease. Proc. Natl. Acad. Sci. USA 100, 2041-2046 (2003). 